\title{
Severe hypercalcemia and a pelvic brown tumor in an adolescent with primary hyperparathyroidism: a case report
}

\author{
O. Legault', M. Inman', N. Moolman², S. Wiebe ${ }^{3}$ A. Poulin ${ }^{4}$ and M. A. Nour ${ }^{1 *}$
}

\begin{abstract}
Background: Primary hyperparathyroidism may present in a myriad of manners, varying from an incidental asymptomatic biochemical finding to gastrointestinal, psychiatric, renal, and bone manifestations. While hyperparathyroidism remains a rare diagnosis in the pediatric population, the initial approach to diagnosis and management of hypercalcemia in children is imperative for the general pediatrician. Herein, we describe an adolescent who presented with a lytic bone lesion and severe, symptomatic hypercalcemia due to primary hyperparathyroidism.
\end{abstract}

Case presentation: A 14-year-old male presented with vomiting, constipation, abdominal pain, and lethargy. He had an elevated total corrected calcium of $4.3 \mathrm{mmol} / \mathrm{L}$. He was found to have a large pelvic lytic tumor consistent with a brown tumor due to primary hyperparathyroidism. He received pharmacologic therapy for stabilization of his hypercalcemia, including intravenous saline, intravenous bisphosphonates, and calcitonin. He subsequently received definitive therapy via parathyroidectomy and his post-operative course was complicated by hungry bone syndrome. Long-term follow-up has found full resolution of the lytic lesion and restored calcium homeostasis.

Conclusions: We present this case to highlight the possible presentations of hypercalcemia and hyperparathyroidism that are essential for a general pediatrician to recognize to ensure prompt diagnosis and management. Evaluation for hypercalcemia should be considered in patients with suggestive symptoms and physical exam findings. To our knowledge, this patient represents the first reported pediatric case of a pelvic brown tumor in an adolescent. While the multi-systemic complications of hyperparathyroidism may be quite severe, swift and appropriate management may mitigate these clinical outcomes.

Keywords: Hyperparathyroidism, Osteitis fibrosa cystica, Hypercalcemia, Hungry bone syndrome, Case report

\section{Background}

Primary hyperparathyroidism is a rare entity in the pediatric population. The presentation is commonly an incidental biochemical finding with asymptomatic hypercalcemia. If symptomatic, patients may describe fatigue, headache, nausea, abdominal pain, vomiting and

\footnotetext{
* Correspondence: munier.nour@usask.ca

'Department of Pediatrics, College of Medicine, Royal University Hospital, University of Saskatchewan, 103 Hospital Drive, SK S7N 0W8 Saskatoon, Canada

Full list of author information is available at the end of the article
}

polydipsia. End organ complications are also described, including nephrocalcinosis, renal impairment and osteoporosis.[1] Bone manifestations of hyperparathyroidism typically present as a silent preferential loss of bone at cortical sites with reduced bone mineral density and osteoporosis, while osteitis fibrosa cystica or 'brown tumors' are a rare manifestation.[2] We report here the case of an adolescent male who presented with severe, symptomatic hypercalcemia due to primary hyperparathyroidism and a large lytic lesion of the pelvis consistent with a brown tumor. This represents, to our

(c) The Author(s). 2020 Open Access This article is licensed under a Creative Commons Attribution 4.0 International License, which permits use, sharing, adaptation, distribution and reproduction in any medium or format, as long as you give appropriate credit to the original author(s) and the source, provide a link to the Creative Commons licence, and indicate if changes were made. The images or other third party material in this article are included in the article's Creative Commons licence, unless indicated otherwise in a credit line to the material. If material is not included in the article's Creative Commons licence and your intended use is not permitted by statutory regulation or exceeds the permitted use, you will need to obtain permission directly from the copyright holder. To view a copy of this licence, visit http://creativecommons.org/licenses/by/4.0/ The Creative Commons Public Domain Dedication waiver (http://creativecommons.org/publicdomain/zero/1.0/) applies to the data made available in this article, unless otherwise stated in a credit line to the data. 
knowledge, the first reported pelvic brown tumor in a pediatric patient. This case highlights the spectrum of disease severity and complications of hyperparathyroidism for the general pediatrician to be mindful of. The principal aspects of the pharmacological and surgical treatment are also be reviewed.

\section{Case presentation}

\section{Clinical Presentation}

A 14-year-old previously healthy male was admitted with a 3-week history of daily emesis, constipation, and lethargy. He had a decreased appetite and $3 \mathrm{~kg}$ weight loss in the preceding weeks. He described vague periumbilical pain and his family noticed a recent depressive change in his mood, including a fatigued appearance, low mood, and withdrawn behaviour. He had no joint or bone pain and denied fever or night sweats. Past medical history was unremarkable. He was on no medications. The family had recently immigrated from the Philippines one-week prior to the onset of symptoms. On exam, he appeared unwell and cachectic with a weight of $37 \mathrm{~kg}$ (-2.1 standard deviation (S.D.)) and a body mass index (BMI) of $14.1 \mathrm{~kg} / \mathrm{m}^{2}$ (<-3 S.D.). He was alert, oriented and cooperative. Neurological exam was unremarkable with normal deep tendon reflexes, bilateral flexor plantar reflexes, a normal gait and normal strength. A large, irregular, tender, mobile abdominal mass of $10 \times 5 \mathrm{~cm}$ was palpable in the left lower quadrant.

\section{Diagnostic Assessment}

Initial laboratory testing demonstrated severe hypercalcemia and hypophosphatemia, with a corrected total serum calcium of $4.30 \mathrm{mmol} / \mathrm{L} \quad(\mathrm{N}: 2.14-$ $2.65 \mathrm{mmol} / \mathrm{L})$ and a phosphate of $0.58 \mathrm{mmol} / \mathrm{L}(\mathrm{N}$ : 0.74-1.52 $\mathrm{mmol} / \mathrm{L})$. Initial serum intact parathyroid hormone $(\mathrm{PTH})$ was elevated at $51.70 \mathrm{pmol} / \mathrm{L}(\mathrm{N}$ : $1.30-6.80 \mathrm{pmol} / \mathrm{L}$ ), later peaking at $70.2 \mathrm{pmol} / \mathrm{L}$. Additional biochemical evaluation on presentation is provided in Table 1.

Table 1 Summary of biochemical investigations at presentation

\begin{tabular}{lll}
\hline Test & Result & Reference Interval \\
\hline Total Calcium (mmol/L) & 4.13 & $2.14-2.65$ \\
Corrected Calcium (mmol/L) & 4.30 & $2.14-2.65$ \\
Phosphate (mmol/L) & 0.58 & $0.74-1.52$ \\
Magnesium (mmol/L) & 0.75 & $0.66-1.07$ \\
Albumin (g/L) & 34 & $35-52$ \\
Alkaline Phosphatase (U/L) & 328 & $40-150$ \\
PTH (pmol/L) & 51.7 & $1.3-6.8$ \\
25-OH Vitamin D (nmol/L) & 45.4 & $70-250$ \\
1,25(OH) $)_{2}$ Vitamin D (pmol/L) & 197 & $64-226$ \\
\hline
\end{tabular}

PTH Parathyroid hormone level
Given the palpable mass and reported symptoms of emesis and constipation, a plain film abdominal radiograph was performed on admission to evaluate the abdominal mass and assess for possible obstruction. This identified large fecal loading of the colon and a lytic lesion within the left iliac wing (Fig. 1). Subsequent computed tomography (CT) and magnetic resonance imaging (MRI) imaging showed the lesion arising within the bone, measuring $13 \times 5.1 \times 5.8 \mathrm{~cm}$ and a scalloped appearance with a T2 intense soft tissue component slightly projecting beyond the anterior table of the iliac bone (Fig. 2). Thyroid ultrasound and Nuclear Medicine technetium $99 \mathrm{~m}$ Sestamibi scan were consistent with the identification of a solitary left superior parathyroid adenoma (Fig. 3).

No further bony lesions were identified with full body skeletal survey radiographs and scintigraphy. Renal ultrasound was unremarkable with no nephrocalcinosis nor nephrolithiasis. Bone mineral densitometry (BMD) demonstrated lumbar spine, whole body and left distal forearm Z-scores of -0.2, -1.1, and 1.7 , respectively.

CT guided core needle biopsy of the iliac bone lesion was performed and underwent histological assessment demonstrating viable bone surrounded by moderately

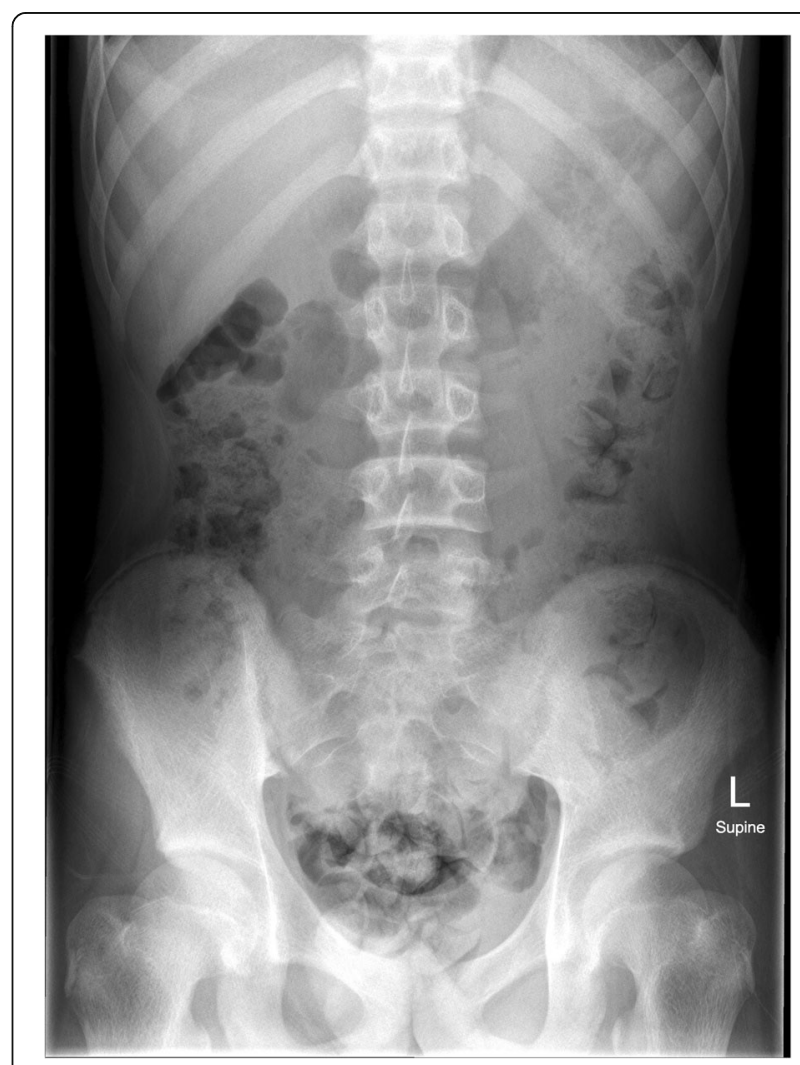

Fig. 1 Radiograph at presentation demonstrating the well-defined low-density lesion (Brown Tumor) within the left iliac bone 


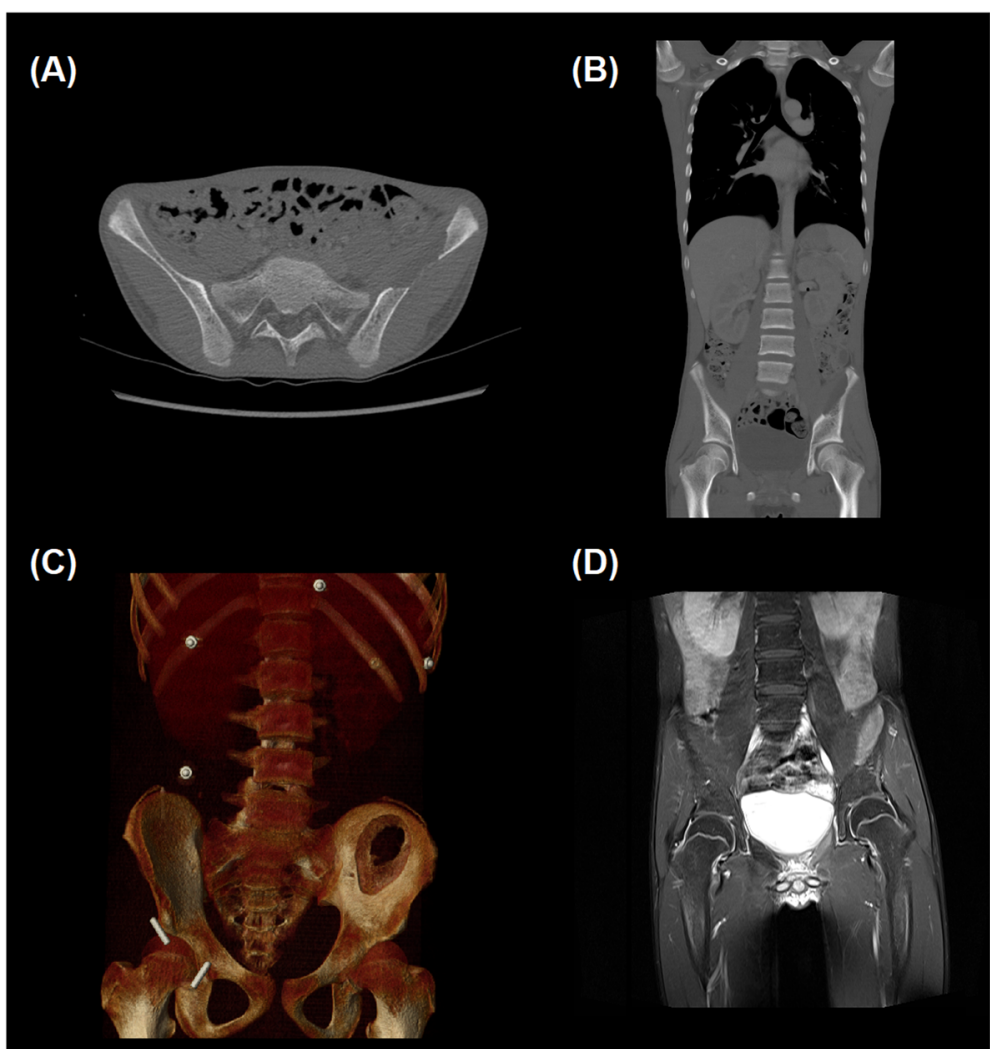

Fig. 2 a Axial, b Coronal (bone window), and c 3-dimensional reconstruction CT imaging demonstrating a lytic lesion in the left iliac bone. d Coronal T2 MR image demonstrating the increased T2 signal in the tissue within the lytic bone lesion

cellular spindle cells with hemosiderin, hemorrhage, and rare osteoclast-like giant cells, consistent with osteitis fibrosa cystica (Fig. 4).

\section{Therapeutic Intervention}

Prior to surgical treatment, hypercalcemia was treated with intravenous (IV) normal saline, multiple doses of subcutaneous (SC) calcitonin and a single dose of IV Zoledronic acid $(0.025 \mathrm{mg} / \mathrm{kg})$. The patient underwent surgical excision of a left superior parathyroid gland $(2.0 \times$ $1.6 \times 0.4 \mathrm{~cm} ; 0.9 \mathrm{~g}$ ) with histology consistent with a parathyroid adenoma (Fig. 5). The postoperative course was complicated by prolonged, recurrent hypocalcaemia with a total serum calcium nadir of $1.98 \mathrm{mmol} / \mathrm{L}$, consistent with "hungry bone syndrome". He required IV and oral replacement of calcium for 6 days. He was later discharged to home with oral supplementation of calcium carbonate and cholecalciferol.

\section{Follow-up and outcomes}

In the subsequent 8 months post-parathyroidectomy, his weight increased $17 \mathrm{~kg}$ to $54 \mathrm{~kg}(-0.4$ S.D.) and BMI to $19.8 \mathrm{~kg} / \mathrm{m}^{2}$ (0.0 S.D.). Serum biochemistry was maintained with gradual weaning and discontinuation of supplementation of calcium and vitamin $\mathrm{D}$; serum total calcium $2.43 \mathrm{mmol} / \mathrm{L}$, phosphate $1.66 \mathrm{mmol} / \mathrm{L}$, and $\mathrm{PTH}$ $6.2 \mathrm{pmol} / \mathrm{L}$. Repeat pelvic MRI within 12-months postparathyroidectomy demonstrated complete resolution of pelvic lesion.

\section{Discussion and conclusions}

Hypercalcemia is a relatively rare presentation in the pediatric population, however, an approach to the differential diagnosis and awareness of initial management steps is important for the general pediatrician. While hypercalcemia in neonates and infants includes a number of genetic and iatrogenic possibilities, the diagnosis in the adolescent - much like in adults - is most commonly due to one of three aetiologies: hyperparathyroidism, malignancy or immobilization.[3] Given the large palpable abdominal mass found in our patient, initial assessments were highly suspicious for an underlying malignancy. However, biochemical and radiologic evaluation confirmed the diagnosis of hyperparathyroidism and proved the abdominal mass to be due to constipation and fecal impaction secondary to severe hypercalcemia. 


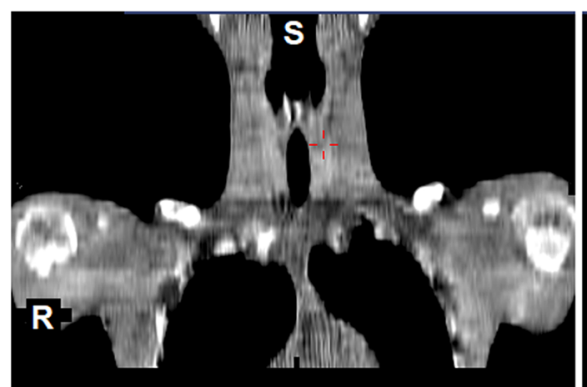

s

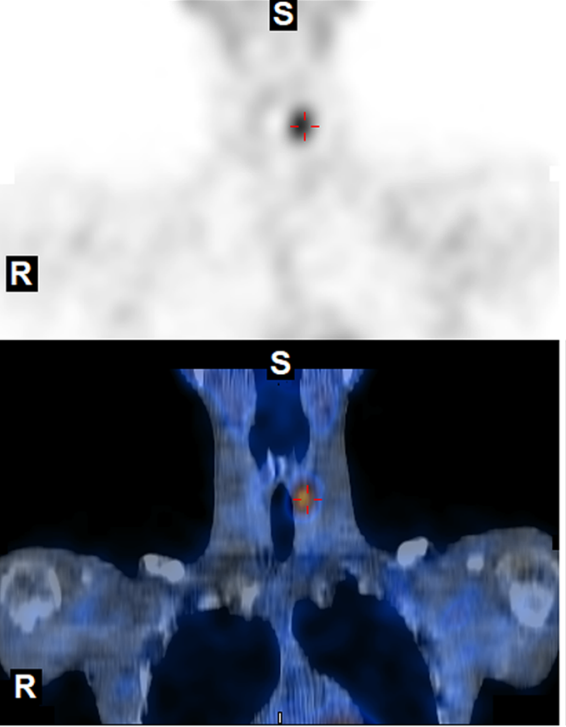

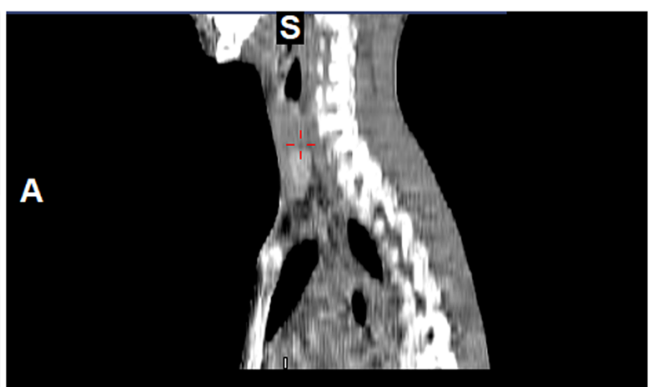

s

A

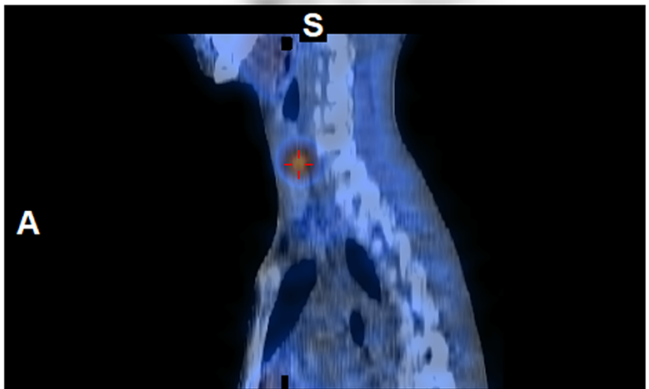

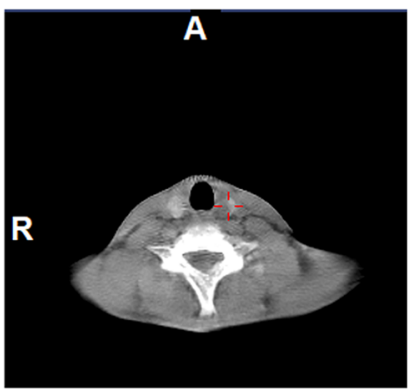

A
$\mathbf{R}$

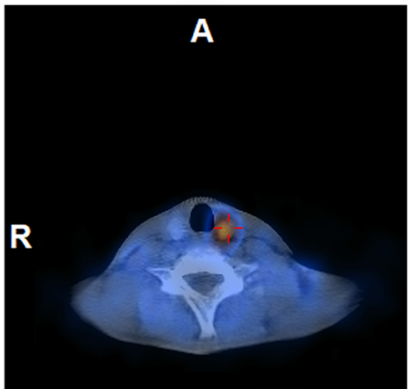

Fig. 3 Nuclear Medicine technetium 99 m Sestamibi parathyroid scan with supplemental SPECT-CT imaging demonstrating a solitary focus of increased radiotracer uptake. From top to bottom, the rows of images demonstrate CT images, images reconstruction with attenuation correction (IRAC), and hybrid CT-IRAC SPECT-CT images, respectively. From left to right, the columns of images depict coronal, sagittal, and transaxial images, respectively. (A: anterior; $R$ : right; $S$ : superior)

Initial management for all patients with severe symptomatic hypercalcemia must begin with IV fluid administration for dilution and subsequent renal excretion of excess calcium. Hypercalcemia can induce dehydration through impaired oral intake and is compounded by possible development of nephrogenic diabetes insipidus. Additional therapies to reduce serum calcium levels may include antiresorptive agents (i.e. bisphosphonates), calcitonin, forced calciuresis, and corticosteroids. Bisphosphonates inhibit osteoclastic activity and hinder hydroxyapatite breakdown,

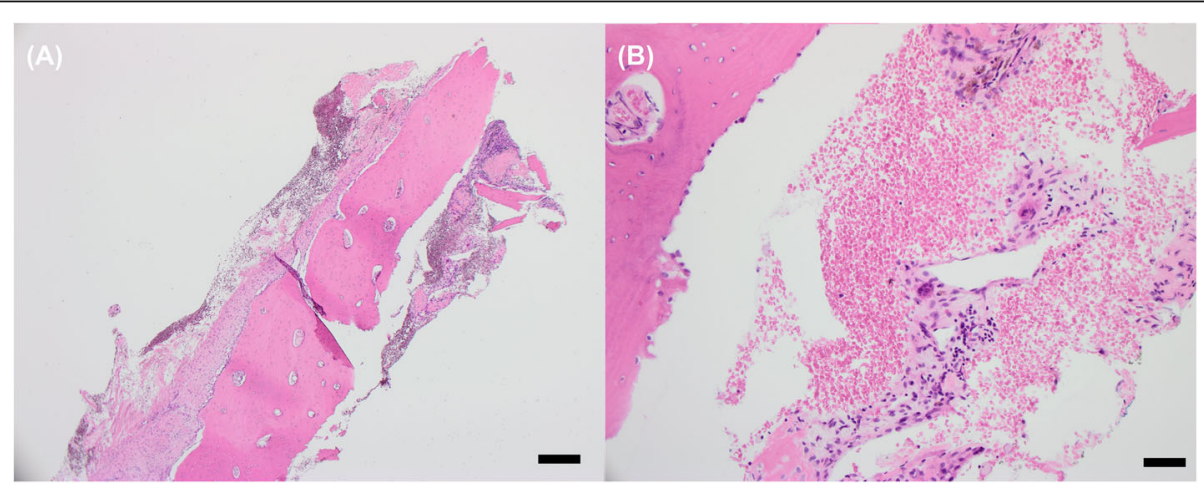

Fig. 4 Core needle biopsy of left iliac crest. a Viable bone with reparative changes at H\&E 4 X magnification. (Black bar denotes $500 \mu m$ at this resolution) $\mathbf{b}$ Rare osteoclast-like giant cells, hemorrhage, and hemosiderin are present in a background of spindle cells consistent with brown tumor at H\&E 20X magnification. (Black bar denotes $50 \mu \mathrm{m}$ at this resolution) 

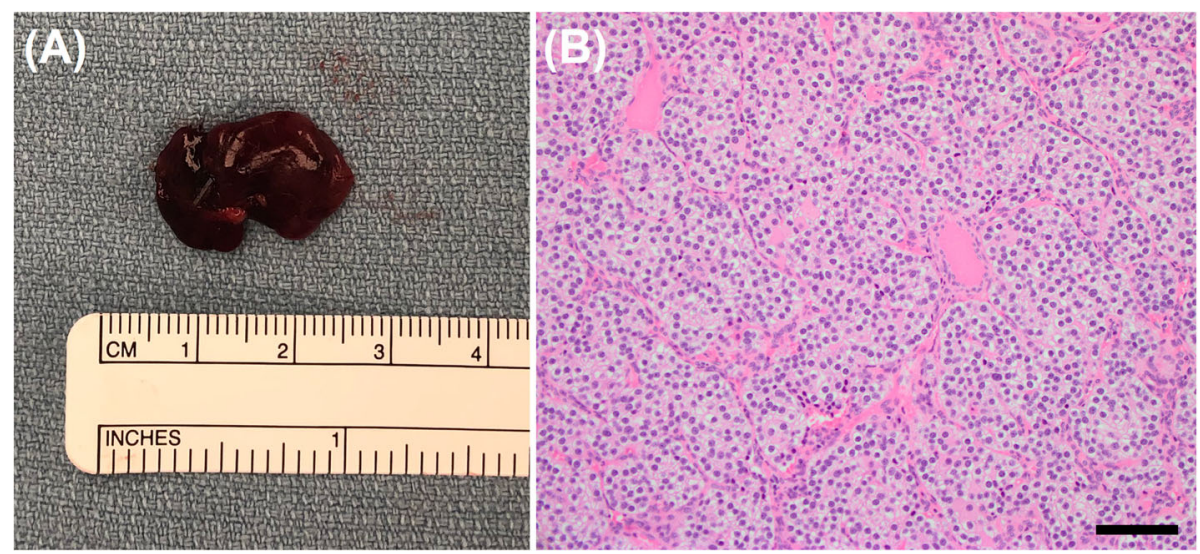

Fig. 5 a Excised left superior parathyroid gland adenoma measuring $2.0 \times 1.6 \times 0.4 \mathrm{~cm}$. b Histological specimen demonstrating nests of proliferating parathyroid cells lacking intermixed adipose tissue, consistent with a parathyroid adenoma (H\&E 20X magnification, black bar denotes $100 \mu \mathrm{m})$

a mineral composed of calcium and phosphate. Calcitonin inhibits bone resorption and forces renal excretion of calcium, but its effect is limited due to development of tachyphylaxis within days of initial exposure. Facilitating calciuresis through diuretic use, such as furosemide, should be delayed until intravascular volume has been replaced. The use of corticosteroids is potentially beneficial in select etiologies of hypercalcemia that are primarily mediated by excessive production of activated vitamin D (ex. granulomatous diseases).

This patient highlights several unique aspects to the presentation of hyperparathyroidism. Primary hyperparathyroidism is characterized by the excessive secretion of PTH by one or multiple parathyroid glands. PTH promotes bone catabolism of cortical bone and bone anabolism of cancellous bone.[4] This was demonstrated here with a relative reduction in BMD of the distal 1/3 forearm - a primarily cortical site - and minimal impact on BMD of the lumbar spine - predominantly trabecular. Osteolytic lesions such as brown tumors manifest in severe and prolonged courses of hyperparathyroidism and excessive osteoclastic activity. Renal involvement from hyperparathyroidism is more frequent than bone involvement and manifests with hypercalciuria, nephrocalcinosis, and nephrolithiasis. Neuropsychological symptoms are also associated with hypercalcemia and include nonspecific complaints such as weakness, fatigue, depression and anxiety as well as complaints of decreased memory and concentration, loss of initiative, irritability, and disturbed sleep.[5].

The diagnosis of a brown tumor is primarily clinical through the presence of either a single or multiple welldemarcated osteolytic lesions in the setting of hyperparathyroidism and hypercalcemia. Histologic features of the tumor are non-specific and may resemble other giant cell tumors $[6,7]$ and is the result of an increased activity of osteoclasts replacing bone with reactive fibrous tissue.[8]
Brown tumors received their name due to the brown tissue appearance resulting from microhemorrhages of interstitium, causing hemosiderin deposits. The incidence of brown tumors are quite rare, occurring in approximately $2 \%$ of adult patients suffering from any form of hyperparathyroidism and commonly affect the mandible, clavicle, ribs, and pelvis.[9-11]. While most presentations of hyperparathyroidism are asymptomatic in occidental countries, skeletal manifestations are seen more commonly in countries such as India, Iran, Saudi Arabia and Thailand.[12] Reports of brown tumors are exceedingly rare in the pediatric patient and the few cases described were reported in children 14 to 18 years of age with osteolytic lesions presenting in the skull, mandibula, scapula, clavicles, ribs, sternum, vertebrae, and long bones. [13-17] To our knowledge a pelvic manifestation of brown tumor has never been reported in a pediatric patient, however cases have been reported in adults 39 to 69 years of age. [11, 18]

Definitive therapy for primary hyperparathyroidism and brown tumors is surgical through parathyroidectomy. In cases of severe, prolonged hyperparathyroidism, there is a significant postoperative risk of developing hungry-bone syndrome.[19] This results in acute hypocalcemia from rapid deposition of calcium into bone. As this hypocalcemia is driven by autonomous 'hungry' osteocytes directing osteoblastic deposition of calcium, the mainstay of therapy for hungry-bone syndrome is replacement of calcium via IV and oral routes. Bisphosphonate therapy was also utilized in order to potentially reduce the expected risk of post-operative hungry bone syndrome.[20] Given bisphosphonates primarily inhibit osteoclastic bone resorption, the mechanism of prevention of hungry bone syndrome is poorly understood, however is thought to be the result of a transient inhibition of bone mineralization. [21] Therapy may further be augmented through the use of active metabolites of Vitamin-D (Calcitriol) to maximize enteric absorption of calcium. [19, 22] 


\section{Conclusions}

With a wide spectrum of presenting features, severe hyperparathyroidism may result in significant pathology, multisystemic involvement with end-organ damage requiring urgent recognition and therapy. Its mimicry of malignancy requires a thorough clinical review and for patients presenting with severe disease, a combination of acute pharmacologic treatment to reduce hypercalcemia followed by definitive surgical intervention are required for optimization of short- and long-term outcomes. This case reports an extremely rare manifestation of chronic hyperparathyroidism, pelvic brown tumor, and exemplifies the importance of clinical recognition of hypercalcemic symptoms to facilitate prompt diagnosis and acute management.

\section{Abbreviations}

BMI: Body mass index; CT: Computed tomography; IV: Intravenous; MRI: Magnetic resonance imaging; PTH: Parathyroid hormone; S.D.: Standard deviation

\section{Acknowledgements}

Not applicable.

\section{Authors' contributions}

$\mathrm{OL}$ and MN performed data collection, manuscript conceptualization, drafted the initial manuscript, and reviewed and revised the manuscript. MI and NM assisted with data collection, manuscript conceptualization, and reviewed and revised the manuscript. SW assisted with data collection, provided interpretation for relevant radiologic examinations, reviewed, and revised the manuscript. AP assisted with data collection, provided interpretation for relevant histologic specimens, reviewed, and revised the manuscript. All authors read and approved the final manuscript as submitted and agree to be accountable for all aspects of the work.

\section{Funding}

None.

\section{Availability of data and materials}

Not applicable.

\section{Ethics approval and consent to participate}

The REB of the University of Saskatchewan approved this case report.

\section{Consent for publication}

Written consent was obtained from the parents of the patient and verbal assent was obtained from the patient. The consent for publication of the attached images, even if entirely unidentifiable, was also obtained.

\section{Competing interests}

The authors declare that they have no competing interests.

\section{Author details}

${ }^{1}$ Department of Pediatrics, College of Medicine, Royal University Hospital, University of Saskatchewan, 103 Hospital Drive, SK S7N 0W8 Saskatoon, Canada. ${ }^{2}$ Department of Surgery, College of Medicine, University of Saskatchewan, 103 Hospital Drive, S7N OW8 Saskatoon, SK, Canada. ${ }^{3}$ Department of Medical Imaging, College of Medicine, University of Saskatchewan, 103 Hospital Drive, S7N OW8 Saskatoon, SK, Canada.

${ }^{4}$ Department of Pathology, College of Medicine, University of Saskatchewan, 103 Hospital Drive, S7N OW8 Saskatoon, SK, Canada.

Received: 30 August 2020 Accepted: 25 November 2020

Published online: 05 December 2020

\section{References}

1. Kollars J, Zarroug AE, van Heerden J, Lteif A, Stavlo P, Suarez L, et al. Primary hyperparathyroidism in pediatric patients. Pediatrics. 2005;115:974-80.
2. Walker MD, Silverberg SJ. Primary hyperparathyroidism. Nat Rev Endocrinol. 2018;14:115-25.

3. Auron A, Alon US. Hypercalcemia: a consultant's approach. Pediatr Nephrol. 2018:33:1475-88.

4. Khan AA, Hanley DA, Rizzoli R, Bollerslev J, Young JEM, Rejnmark L, et al. Primary hyperparathyroidism: review and recommendations on evaluation, diagnosis, and management. A Canadian and international consensus. Osteoporos Int. 2017;28:1-19.

5. Silverberg SJ, Clarke BL, Peacock M, Bandeira F, Boutroy S, Cusano NE, et al. Current issues in the presentation of asymptomatic primary hyperparathyroidism: proceedings of the Fourth International Workshop. J Clin Endocrinol Metab. 2014;99:3580-94.

6. Shetty AD, Namitha J, James L. Brown Tumor of Mandible in Association with Primary Hyperparathyroidism: A Case Report. J Int Oral Health. 2015;7:50-2.

7. Rosenberg AE, Nielsen GP. Giant cell containing lesions of bone and their differential diagnosis. Current Diagnostic Pathology. 2001;7:235-46.

8. Mori H, Okada Y, Arao T, Shimaziri S, Tanaka Y. A case of multiple brown tumors with primary hyperparathyroidism. J Bone Miner Metab. 2013;31:123-7.

9. Agnihotri M, Kothari K, Naik L. $\Omega$ Brown tumor of hyperparathyroidism. Diagn Cytopathol. 2017;45:43-4.

10. Panagopoulos A, Tatani I, Kourea HP, Kokkalis ZT, Panagopoulos K, Megas P. Osteolytic lesions (brown tumors) of primary hyperparathyroidism misdiagnosed as multifocal giant cell tumor of the distal ulna and radius: a case report. J Med Case Rep. 2018;12:176.

11. Glushko T, Banjar SSA, Nahal A, Colmegna I. Brown tumor of the pelvis. Cleve Clin J Med. 2015:82:799-800.

12. Bandeira F, Cassibba S. Hyperparathyroidism and Bone Health. Curr Rheumatol Rep. 2015;17:48.

13. Atabek ME, Pirgon O, Sert A, Esen HH. Extensive brown tumors caused by parathyroid adenoma in an adolescent patient. Eur J Pediatr. 2008;167:117-9.

14. Eklioglu BS, Atabek ME, Akyürek N. Parathyroid adenoma presented with multiple brown tumors and nephrocalcinosis. J Pediatr Endocrinol Metab. 2013:26:213-4.

15. Satpathy AS, Dasgupta A, Dutta C, Mohan NVK, Satpathy S. Osteitis fibrosa cystica of mandible in hyperparathyroidism-jaw tumor syndrome: A rare presentation and review of literature. Natl J Maxillofac Surg. 2017;8:162-6.

16. Aslan S, Bilgici MC, Bernay RF, Aydin HM, Selcuk MB. Parathyroid adenoma presenting with multiple Brown tumors in an adolescent patient. North Clin Istanb. 2018;5:361-4.

17. Lando HM. A case report of a patient with hyperparathyroidism and presumed sellar/parasellar brown tumor. Endocr Regul. 2012;46:31-6.

18. Radulescu D, Chis B, Donca V, Munteanu V. Brown tumors of the femur and pelvis secondary to a parathyroid carcinoma: report of one case. Rev Med Chil. 2014;142:919-23.

19. Witteveen JE, van Thiel S, Romijn JA, Hamdy N a. T. Hungry bone syndrome: still a challenge in the post-operative management of primary hyperparathyroidism: a systematic review of the literature. Eur J Endocrinol. 2013;168:R45-53.

20. Mayilvaganan S, Vijaya Sarathi HA, Shivaprasad C. Preoperative zoledronic acid therapy prevent hungry bone syndrome in patients with primary hyperparathyroidism. Indian J Endocrinol Metab. 2017;21:76-9.

21. Adamson BB, Byars J, Boyce BF, Gallacher SJ, Ralston SH, Boyle IT, et al. Mineralisation defects with pamidronate therapy for Paget's disease. The Lancet. 1993;342:1459-60.

22. Agarwal G, Mishra SK, Kar DK, Singh AK, Arya V, Gupta SK, et al. Recovery pattern of patients with osteitis fibrosa cystica in primary hyperparathyroidism after successful parathyroidectomy. Surgery. 2002;132: 1075-83. discussion 1083-1085.

\section{Publisher's Note}

Springer Nature remains neutral with regard to jurisdictional claims in published maps and institutional affiliations. 